\title{
Microtacticity and Mean Length of Sequences of the Stereospecific Polymers Obtained by the Control of Both Symmetric and Asymmetric Sites
}

\author{
Riichirô CHÛJô \\ Department of Polymer Chemistry, Tokyo Institute of Technology, \\ 12-1, Ookayama 2-chome, Meguro-ku, Tokyo 152, Japan.
}

(Received August 28, 1974)

KEY WORDS Stereospecific Polymerization / Symmetry / Asymmetry / Tacticity / Mean Length / Ising Model /

The Ising model ${ }^{1}$ has been widely accepted as a tool for analysis of the configuration and conformation of high polymers. The applications of this model have been extended to both synthetic and biological polymers. Among these works, the application to the configuration or the propagation mechanism in stereospecific polymers can be divided into two classes: one is for the polymers obtained by the control of symmetric sites and the other is for those by the control of asymmetric sites. In the former, two-valued logical variables are either isotactic or syndiotactic additions; in the latter they are optical, either $d$ or $l$ selection. Many papers have been published for both classes since Miyake and Chûjô, ${ }^{2}$ reported on the former type of control, and Shelden and his colleagues on the latter type. $^{3}$ As a natural extension of these papers, there have also been papers ${ }^{4,5}$ which are related to polymers obtained by the control of both sites. These polymers are of great interest, because their microtacticities (fractions of isotactic dyad and so on) and the mean lengths of sequences behave in different manners with the changes of energetic parameters. ${ }^{6}$ The purpose of this communication is to give a unified description of such polymers.

Two energetic parameters are introduced here: one is the difference of activation free energy per monomer required for syndiotactic addition from that for isotactic addition (hereafter this is denoted by $U$ ), and the other is the difference between activation free energies required for optical $d$ and $l$ selections, which is denoted by
$E$ (the results are independent of the selection of the sign for $E$ ). If one of the optical configurations is specified by $\alpha$ and the other by $\beta$, and if the Lebesgue measure for an addition of $\beta$ monomer onto $\alpha$ monomeric unit in the propagation end is specified by $P_{\alpha \beta}$, the transition matrix

$$
\begin{aligned}
\mathbf{P} & =\left(\begin{array}{l}
P_{\alpha \alpha} P_{\alpha \beta} \\
P_{\beta \alpha} P_{\beta \beta}
\end{array}\right) \\
& =\left(\begin{array}{ll}
\exp \{(U-E) / 2 k T\} & \exp \{-(U-E) / 2 k T\} \\
\exp \{-(U+E) / 2 k T\} & \exp \{(U+E) / 2 k T\}
\end{array}\right)
\end{aligned}
$$

is obtained by the usual method in the application of Ising model. In this equation $k$ and $T$ denote the Boltzmann constant and the polymerization temperature, respectively. Furthermore, it should be noted that eq 1 is valid for the system in which both the symmetric and the asymmetric stochastic processes obey the Bernoulian trial. The extension of eq 1 to the Markovian process is rather simple, but such an extension is not taken into account here because of the ambiguities expected to occur in the analysis of experimental data due to the increase in the number of controllable parameters. The normalization factor $Q$, which corresponds to the statistical-mechanical partition function of the equilibrium system, is given by the trace of matrix and it can be written by

$$
Q=\text { Trace }\left(\mathbf{P}^{N}\right)=\mu^{+N}+\mu^{-N}
$$

where $N$ is the degree of polymerization of the polymer considered, and $\mu^{+}$and $\mu^{-}$are the two eigenvalues of matrix $\mathbf{P}$. The observable 
quantities are not the fractions of the optical isotactic dyad, $\theta_{i}$, must be obtained explicitly configurations but those of the isotactic and as a measure of microtacticity from eq 1 . This syndiotactic ones, in experiments such as nuclear can be easily done by the usual method and magnetic resonance. Therefore, the fraction of we have

$$
\theta_{\mathrm{i}}=\frac{1}{2}\left(1-\frac{1}{N} \frac{\partial \ln Q}{\partial(U / 2 k T)}\right)=1-\frac{\exp \frac{2 U}{k T}}{\left(\sinh ^{2} \frac{E}{2 k T}+\exp \frac{2 U}{k T}\right)^{1 / 2}+\sinh ^{2} \frac{E}{2 k T}+\exp \frac{2 U}{k T}}
$$

The fraction of syndiotactic dyad can also be obtained by

$$
\theta_{\mathrm{s}}=1-\theta_{\mathrm{i}}
$$

We can easily obtain the contours of isotacticity on the $U-E$ plane with the aid of numerical computation. Numerical computations were carried out by HITAC-8700 system at the Information Processing Center, Tokyo Institute of Technology. A part of the results for $300 \mathrm{~K}$ is shown in Figure 1.

The mean length of isotactic sequences, $\lambda_{i}$, is obtained by a probabilistic consideration and we have

and we have also

$$
\lambda_{i}=\frac{\sum_{n=1}^{\infty} n P_{\alpha} P_{\alpha \beta} P_{\beta \beta}^{n} P_{\beta \alpha}+\sum_{n=1}^{\infty} n P_{\beta} P_{\beta \alpha} P_{\alpha \alpha}^{n} P_{\alpha \beta}}{\sum_{n=1}^{\infty} P_{\alpha} P_{\alpha \beta} P_{\beta \beta}^{n} P_{\beta \alpha}+\sum_{n=1}^{\infty} P_{\beta} P_{\beta \alpha} P_{\alpha \alpha}^{n} P_{\alpha \beta}}
$$

$$
\lambda_{\mathrm{S}}=\frac{\sum_{n=1}^{\infty}(2 n-1) P_{\alpha} P_{\alpha \alpha} P_{\alpha \beta}^{n} P_{\beta \alpha}^{n-1} P_{\beta \beta}+\sum_{n-1}^{\infty} 2 n P_{\alpha} P_{\alpha \alpha} P_{\alpha \beta}^{n} P_{\beta \alpha}^{n} P_{\alpha \alpha}}{+\sum_{n=1}^{\infty}(2 n-1) P_{\beta} P_{\beta \beta} P_{\beta \alpha}^{n} P_{\alpha \beta}^{n-1} P_{\alpha \alpha}+\sum_{n=1}^{\infty} 2 n P_{\beta} P_{\beta \beta} P_{\beta \alpha}^{n} P_{\alpha \beta}^{n} P_{\beta \beta}}
$$

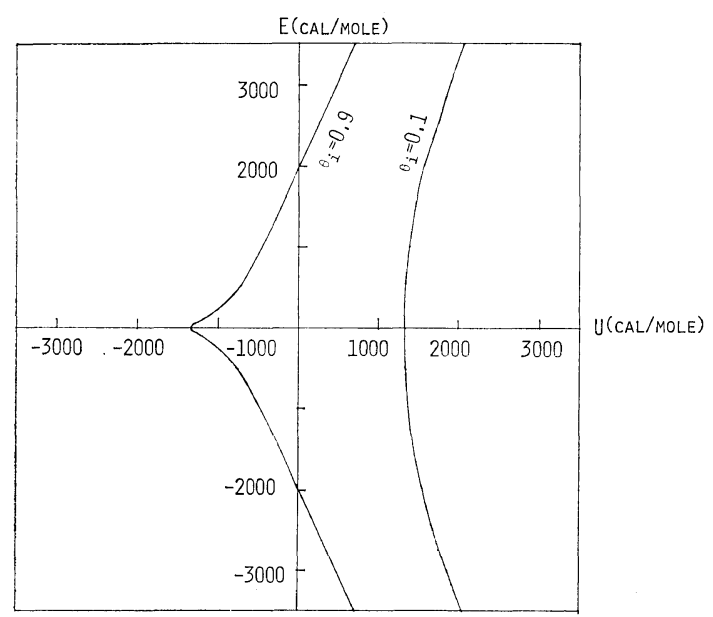

Figure 1. Contours of isotacticity $\theta_{\mathrm{i}}$ at $300 \mathrm{~K}$ on $E-U$ plane.

for the mean length of syndiotactic sequences. In these two equations, $P_{\alpha \beta}$ and other probabilistic parameters are not Lebesgue measures

but probability ones normalized by the division by $P_{\alpha \alpha}+P_{\alpha \beta}$. Continuing the calculations of these two equations, we arrive at these formulae:

$$
\begin{aligned}
\lambda_{\mathrm{i}}= & 4 \mathrm{e}^{U / 2 k T} \cosh \frac{E}{k T} \\
& \times\left\{\mathrm{e}^{E / 2 k T} \operatorname{sech} \frac{U+E}{2 k T}+\mathrm{e}^{-E / 2 k T} \operatorname{sech} \frac{U-E}{2 k T}\right\}^{-1} \\
\lambda_{\mathrm{S}}= & 4 \mathrm{e}^{-U / 2 k T} \\
& \times\left\{\mathrm{e}^{E / 2 k T} \operatorname{sech} \frac{U+E}{2 k T}+\mathrm{e}^{-E / 2 k T} \operatorname{sech} \frac{U-E}{2 k T}\right\}^{-1}
\end{aligned}
$$

The contours from these equations are plotted in Figure 2. Numerical calculations were carried out for the cases in which the curves cross the abscissae at the same positions as shown in Figure 1.

Comparing these two figures, we notice that longer isotactic lengths are generally obtained in 


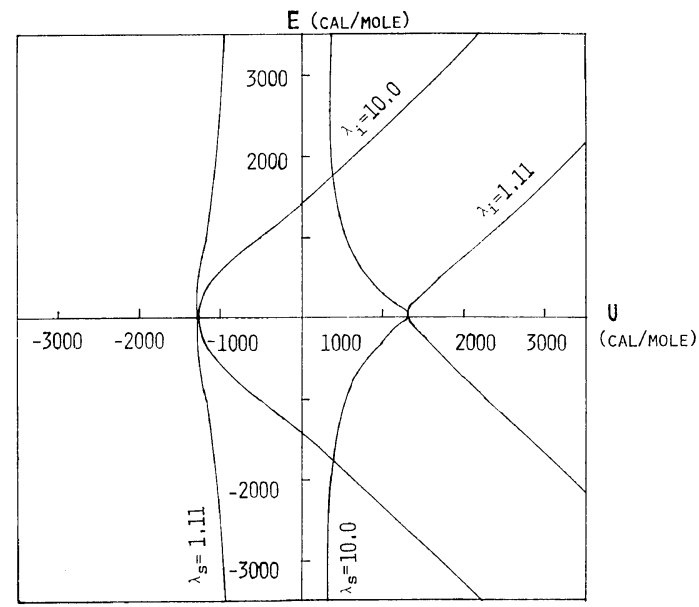

Figure 2. Contours of the mean length of isotactic sequences $\lambda_{i}$ at $300 \mathrm{~K}$ on $E-U$ plane.

the system with a larger contribution from asymmetric sites, even if the microtacticites are equal. A similar figure was previously published by Luisi and Mazo. $^{5}$ In their papers, the sign of $U$ was misused; thus their figure was reproduced in Figure 3 after an inversion around the $E$-axis. They obtained contours from the following criteria:

$$
\begin{array}{lll}
\text { Isotactic: } & \theta_{\mathrm{d}}>0.96, & \lambda_{\mathrm{d}}>25 \\
\text { Syndiotactic: } & \theta_{\mathrm{d}} \simeq 0.5, & \lambda_{\mathrm{d}} \simeq 1 \\
\text { Stereoblock: } & \theta_{\mathrm{d}} \simeq 0.5, & \lambda_{\mathrm{d}}>25
\end{array}
$$

In these criteria, $\theta_{\mathrm{d}}$ denotes the fraction of monad with the same optical configuration and $\lambda_{d}$ the mean length of the sequences with the same optical configuration. As is seen from the comparison of Figure 1 with 2, there does not exist any criterion which is satisfied simultaneously for both microtacticity and mean length. Furthermore, their criteria are biased in favor of either isotactic or syndiotactic polymers. This is inevitable so far as the criteria are defined in terms of the monad. In Figure 3 the intersections of the curves $\theta_{\mathrm{i}}=0.5$ and $\theta_{\mathrm{s}}=0.5$ with the $U$-axis are asymmetric around the $E$-axis with each other. This asymmetry is due to the above mistaken criteria. Such a criticism of the results of Luisi and Mazo is also an objective of the present study.

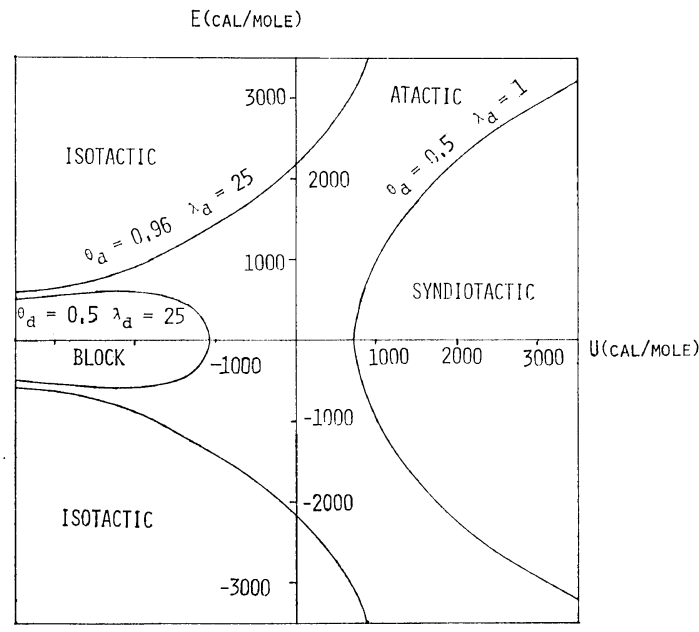

Figure 3. Divisions into five kinds of stereospecific polymers at $300 \mathrm{~K}$ according to the criteria due to Luisi and Mazo. (Reproduced from ref 5 after an inversion around the $E$-axis.)

Acknowledgment. The author would like to express his thanks to Professor P. L. Luisi at Eidgenössische Technische Hochschule Zürich and Professor R. M. Mazo at University of Oregon for their discussions through correspondence. Although it is regrettable that they and the author have not yet come to an agreement, the author has been much stimulated through such discussions. His thanks are also due to Mr. K. Tsuyama of this institute for his kind assistance in the execution of numerical computations. Finally, the author thanks Professor J. Furukawa at Kyoto University for his encouragement.

\section{REFERENCES}

1. E. Ising, Z. Physik, 31, 253 (1925).

2. A. Miyake and R. Chûjô, J. Polym. Sci., 44, 173 (1960).

3. R. A. Shelden, T. Fueno, T. Tsunetsugu, and J. Furukawa, J. Polym. Sci., Part B, 3, 23 (1965).

4. R. A. Shelden, T. Fueno, and J. Furukawa, $J$. Polym. Sci., Part A-2, 7, 763 (1969).

5. P. L. Luisi and R. M. Mazo, J. Polym. Sci., Part A-2, 7, 775 (1969).

6. R. Chûjô, J. Macromol. Sci--Phys., Part B, 2, 1 (1968). 